\title{
Neue Bauweisen in der Intralogistik durch Holzverbundwerk- stoffe - Verbindungselemente für dynamische Belastungen
}

\author{
Dipl.-Ing. Ronny Eckardt, Dipl.-Ing. Sven Eichhorn \\ Technische Universität Chemnitz \\ Institut für Fördertechnik und Kunststoffe \\ Professur Fördertechnik, Arbeitsgruppe Anwendungstechnik erneuerbarer \\ Werkstoffe
}

\begin{abstract}
In der technischen Logistik bieten Bauweisen aus Holzverbundwerkstoffen, speziell aus schichtförmigen aufgebauten Halbzeugen (WVC - Wood-VeneerComposites), eine Alternative zu Konstruktionen aus Metallwerkstoffen, vor allem aufgrund ihrer guten spezifischen mechanischen Eigenschaften sowie ihrer günstigen Korrosionseigenschaften. Benötigte Verbindungsmittel entstammen dabei dem Bau- oder Möbelsektor und sind vorrangig statischen Belastungen angepasst.

Der Vortrag gibt einen kurzen Einblick in Bauweisen mit WVC und stellt dabei Forschungsergebnisse zu statischen und dynamischen Untersuchungen an prinzipiell geeigneten Verbindungselementen in den Vordergrund. Dabei wird vor allem auf holztypische Problemstellungen im Zusammenhang mit dynamischen Belastungen eingegangen. An Funktionsmustern werden mögliche Anwendungen in der Fördertechnik, speziell für den Stückguttransport, dargestellt.
\end{abstract}




\section{$1 \quad$ Einleitung und Motivation}

Holz bzw. Holzwerkstoffe sind traditionelle Konstruktionsmaterialien, die im letzten Jahrhundert, vor allem in technischen Bereichen, schrittweise durch Metalle (z.B. Stahl und Aluminium) verdrängt wurden. Heutzutage beschränkt sich ihre Anwendung vorrangig auf bautechnische Gebiete bzw. Anwendungen im Innenbereich, die in erster Linie statischen Anforderungen unterliegen. Eine zunehmende Ressourcenknappheit führt mittlerweile dazu, dass derartige Werkstoffe wieder verstärkt als Alternative in technische Bereiche Einzug halten. Neben diesen ökologischen Gesichtspunkten zeichnen sich Holzwerkstoffe ebenso durch gute spezifischmechanische Eigenschaften (spezifisch: auf die Werkstoffdichte bezogen), eine gute Resistenz gegenüber vielen Chemikalien sowie ihre hohe Verfügbarkeit als nachwachsender Rohstoff aus. Schlussendlich bieten sie auch aus wirtschaftlicher Sicht Vorteile gegenüber Metallwerkstoffen.

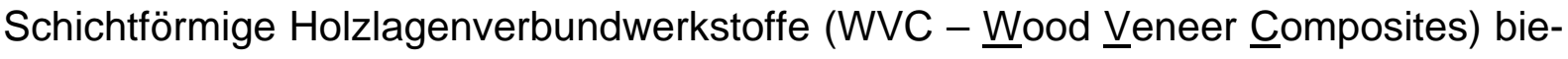
ten infolge ihres Eigenschaftsspektrums die Möglichkeit, zumindest in ausgewählten Teilbereichen des Maschinen- und Anlagenbaus, herkömmliche Konstruktionswerkstoffe zu ersetzen. Über einen Schichtaufbau lassen sich gezielt Eigenschaften steuern. So kann beispielsweise die holztypische Richtungsabhängigkeit der Eigenschaften (Anisotropie) verringert werden. Gleiches trifft auf das Quell- und Schwindverhalten unter Feuchtigkeitseinfluss zu. Auch hier kann durch einen kreuzweise orientierten Lagenaufbau (Beispiel: Sperrholz) die Auswirkung dieses Phänomens minimiert werden.

Da sich das derzeitige Anforderungsspektrum dieser Werkstoffe vorrangig auf statische Bereiche konzentriert, müssen die vorhandenen Kennwerte um dynamische Betrachtungen erweitert werden. Anwendungen im Maschinen- und Anlagenbau, speziell als Gestellsysteme in der technischen Logistik, erfordern zudem die Beherrschung der Verbindungstechnik. Werkstofftypische Eigenheiten, hierbei sind hauptsächlich das rheologische Verhalten der Holzwerkstoffe sowie ihre klimaabhängigen Eigenschaftsschwankungen gemeint, müssen in die Betrachtungen zur Verbindungstechnik einbezogen werden.

Ziel dieses Vortrages ist es, systematisch durchgeführte Untersuchungen an Verbindungsmitteln speziell für Holzwerkstoffe vorzustellen. Hierbei sollen sowohl statische, vor allem aber, für maschinenbautechnische Anwendungen erforderliche, dynamische Betrachtungen aufgezeigt werden. Abschließend werden praktische Anwendungsmöglichkeiten vorgestellt, für die diese Versuche maßgeblich sind. 


\section{Auswahl und Prüfmethodik}

Moderne Logistik zeichnet sich durch ein hohes Maß an Flexibilität aus, wodurch Gestellsysteme eine entsprechende Modularität aufweisen müssen. Verbindungen dieser Gestellkomponenten sollten daher prozesssicher sein, jedoch gleichzeitig einfach lösbar. Dieser Aspekt schließt rein stoffschlüssige Verbindungen, z.B. Kleben oder Schweißen, von vornherein aus. Auch rein formschlüssige Verbindungen sind problematisch, da Holz hygroskopische Eigenschaften besitzt, die unter Feuchtigkeitseinfluss zu zum Teil massiven Formänderungen führen. Eventuelle Passverbindungen sind daher nur bedingt möglich. Somit wurde sich im Rahmen dieses Forschungsprojektes auf kraftschlüssige Verbindungen, teilweise in Kombination mit formschlüssigen, konzentriert.

Holz weist als viskoelastisches Material zeitabhängige mechanische Eigenschaften auf. Speziell die Problematik des Kriechens (Zunahme der Formänderung unter konstanter Last [WS07]) muss bei prozesssicheren Verbindungen in die Betrachtungen einbezogen werden. Sollen Verbindungsmittel genutzt werden, sollten diese möglichst materialschonend in den Grundwerkstoff eingebracht werden. Strukturschädigungen jedweder Art wirken sich bei Holzwerkstoffen wesentlich nachteiliger auf die Gesamtstabilität aus als dies bei Metallen der Fall ist. Weiterhin gilt es zu beachten, dass durch die Verwendung metallischer Verbindungsmittel äußeren Umwelteinflüssen möglichst wenig Angriffsfläche geboten wird und gleichzeitig die Wirtschaftlichkeit der Verbindung, vor allem bei größeren Stückzahlen, noch gewährleistet ist.

Üblicherweise finden stiftförmige Verbindungsmittel verbreitete Anwendung in diesen Bereichen. Eine verfügbare und wirtschaftliche Lösung nach dem Stand der Technik stellt das Einbringen von Einsätzen aus Metall nach DIN 7965 [DIN65] durch Einschrauben oder Einschlagen in die Holzwerkstoff dar. Das Insert ist nur von einer Seite Umwelteinflüssen (z.B. korrosiver Atmosphäre) ausgesetzt und durch das genormte Regelgewinde im inneren Bereich ist die Anbindung über eine konventionelle Verschraubung möglich. Die Schädigung des Holzes ist durch das punktuelle Einbringen der Bohrung vergleichsweise gering. Die Untersuchungen im Forschungsprojekt konzentrierten sich demzufolge darauf, am Markt verfügbare Inserttypen auf ihre Eignung hin zu untersuchen und gegebenenfalls Ansätze für eine Verbesserung dieser Verbindungsmittel aufzuzeigen.

Grundsätzlich erfolgt eine Belastung der Einsätze auf Biegung und Zug (Auszug), im Ergebnis dessen das Insert aus dem Grundwerkstoff herausgerissen wird. Häufiger in der praktischen Anwendung ist das auf Biegung belastetet Teil, ungünstiger und damit Grenzwert ist die Beanspruchung auf Zug [nach DIN EN 1382 [EN99]: Ausziehtragfähigkeit bei Belastungen in Richtung der Längsachse des Verbindungsmittels (Einbringrichtung)]. Entsprechend dieser Anforderungen orientieren sich die Versuchsaufbauten an beiden Belastungsarten. 
a) Biegung

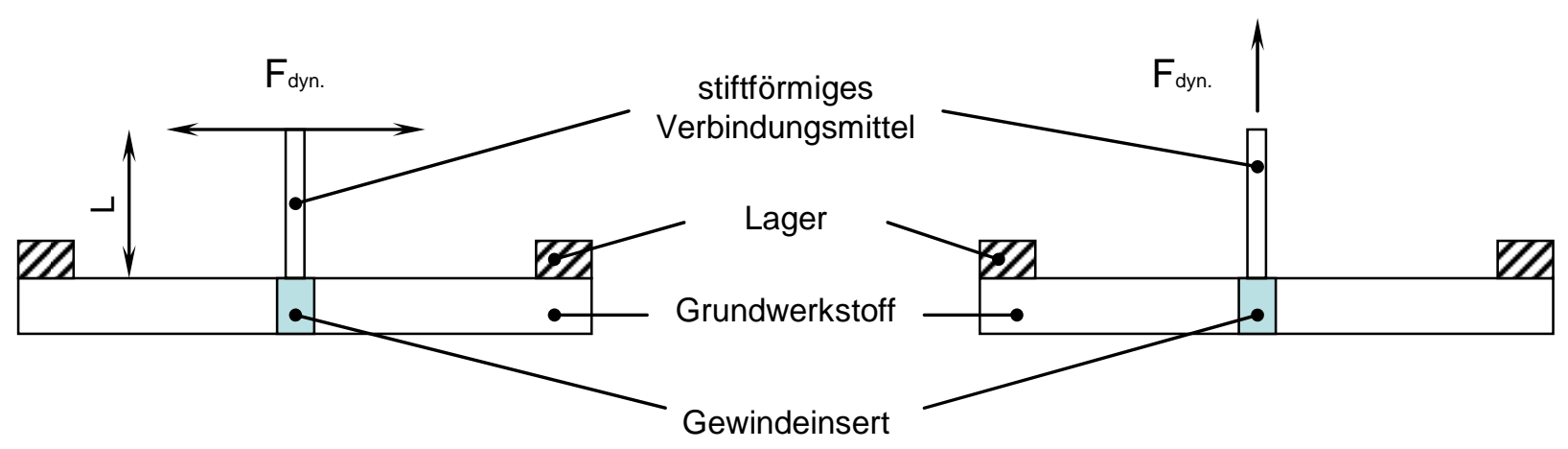

Abbildung 1: Versuchsanordnung der Grundbelastungen (linke Abb.: Biegung, rechte Abb.: Zug)

Die modularen Gestellaufbauten und damit die Verbindungsstellen finden vor allem bei dynamischen Lastkollektiven Anwendung. Das bedeutet, dass der statische bzw. quasistatische Bemessungsfall aus dem Bauwesen um dynamische Betrachtungen erweitert werden muss. Die grundlegende Auswahl und Untersuchung der Verbindungsmittel erfolgte in einem dreistufigen Experiment nach dynamischen Gesichtspunkten.

- Auswahl möglicher Inserttypen durch Charakterisierung der dynamischen Eigenschaften der Verbindungsstelle (dynamische Kurzzeituntersuchungen)

- Gegenüberstellung dynamisch belasteter und unbelasteter Proben im statischen Zugversuch; Untersuchung geometrischer und klimatischer Einflussfaktoren auf die Verbindungsfestigkeit

- Dynamische Langzeituntersuchungen - Finden der dynamischen Grenzbelastung als Bemessungsgrundlage

Im Rahmen dieser Untersuchungen sollen Kenntnisse zum Verbindungsmittel, zum Grundwerkstoff, vor allem aber zu den Wechselwirkungen beider gewonnen werden. Nur eine Verbindungstechnik, die statischen und dynamischen Anforderungen Rechnung trägt, ist zur Verwendung in modularen Gestellsystemen geeignet.

\section{Versuche}

\subsection{Dynamische Kurzzeituntersuchungen}

Ziel der dynamischen Kurzzeituntersuchungen ist die generelle Einordnung bzw. Klassifizierung der ausgewählten Verbindungsmittel (s. Tabelle 1) hinsichtlich ihrer Anwendbarkeit bei höheren dynamischen Lasten. Der grundlegende Versuchsaufbau entspricht dem aus Abbildung 1a. Eine Charakterisierung des dynamischen Verhaltens erfolgte über die Durchführung eines einfachen Löseversuchs. Die zu untersuchenden Inserttypen können der folgenden Tabelle entnommen werden. 
Tabelle 1: Versuchsparameter der dynamischen Kurzzeitversuche

\begin{tabular}{|c|c|c|}
\hline Probe - Verbindungsmittel & Beschreibung & Darstellung \\
\hline $\begin{array}{l}\text { Versuchsreihe } 1 \text {, } \\
\text { Schraubinsert } 1 \text {, } \\
\text { SI } 1\end{array}$ & $\begin{array}{l}\text { metrisches Innengewinde M8 } \\
\text { und Außengewinde nach DIN } \\
7965, \mathrm{~d}=13 \mathrm{~mm} \text {, } \\
\text { Einschraublänge I = } 15 \mathrm{~mm}, \\
\text { (Hersteller: Rampa) }\end{array}$ & \\
\hline $\begin{array}{l}\text { Versuchsreihe } 2, \\
\text { Schraubinsert } 2 \\
\text { SI } 2\end{array}$ & $\begin{array}{l}\text { metrisches Innengewinde M8 } \\
\text { und metrisches Außengewinde } \\
\text { M12, Einschraublänge I = } \\
15 \mathrm{~mm} \\
\text { (Hersteller: Rampa) }\end{array}$ & \\
\hline $\begin{array}{l}\text { Versuchsreihe } 3 \text {, } \\
\text { Einschlagmuffe } 1, \\
\text { EM } 1\end{array}$ & $\begin{array}{l}\text { mit Rändelring, Innengewinde } \\
M 8 \text { und Außendurchmesser } \\
D=10,5 \mathrm{~mm} \text {, Einschraublänge } \\
\mathrm{I}=15 \mathrm{~mm}, \\
\text { (Hersteller: Rampa) }\end{array}$ & \\
\hline $\begin{array}{l}\text { Versuchsreihe } 4, \\
\text { Einschlagmuffe } 2 \text {, } \\
\text { EM } 2\end{array}$ & $\begin{array}{l}\text { mit Zähnen, Innengewinde M8 } \\
\text { und Außendurchmesser } \\
D=15 \mathrm{~mm} \text {, Einschraublänge } \\
\mathrm{I}=15 \mathrm{~mm} \\
\text { (Hersteller: Rampa) }\end{array}$ & \\
\hline $\begin{array}{l}\text { Versuchsreihe } 5, \\
\text { Holzschraube, } \\
\text { HS (Vergleichsuntersuchun- } \\
\text { gen) }\end{array}$ & $\begin{array}{l}\text { Holzschrauben mit Gewinde } \\
\text { nach DIN 7998, D =6,3 mm, } \\
\text { Einschraublänge I = } 15 \mathrm{~mm} \\
\text { (Hersteller: Rampa) }\end{array}$ & \\
\hline $\begin{array}{l}\text { Versuchsreihe 6, } \\
\text { Quergewindebolzen, } \\
\text { QGB } \\
\text { (Vergleichsuntersuchungen) }\end{array}$ & $\begin{array}{l}\text { Quergewindebolzen mit Au- } \\
\text { ßendurchmesser } 12 \mathrm{~mm} \text { und } \\
\text { Innengwinde M8 } \\
\text { (Hersteller: Rampa) }\end{array}$ & \\
\hline
\end{tabular}

Hierbei wurden folgende Versuchsparameter verwendet:

- definierter Probekörper aus 15-lagigem Birkensperrholz (BFU-BU 100); Rohdichte: $0,69 \mathrm{~g} / \mathrm{cm}^{3}$; Lagenausrichtung $0^{\circ} / 90^{\circ}$; Abmessungen, [80x80x20] mm), Holzfeuchte ca. 7,5 \% (Prüfklima ca. $20^{\circ} \mathrm{C}, 50 \%$ rel. Luftfeuchte)

- Versuchsaufbau nach Abbildung 1a (Biegung), Hebelarm 18,5 mm

- wechselnde sinusförmige dynamisch Belastung $\pm 700 \mathrm{~N}$ (kraftgeregelt), bei einer Prüffrequenz von $10 \mathrm{~Hz}$

- Versuche, wenn möglich, bis $10 \mathrm{e} 5$ Lastwechsel (LW), Beurteilung der Verbindung 
Die in den Einspannpunkt (Verschraubung) der Verbindung eingebrachte Belastung erzeugt durch Setzerscheinungen und Zerstörung im Holzwerkstoff eine Verformung, die sich sowohl als Veränderung der Durchbiegung des Hebelarms bei Belastungsbeginn (1 LW) als auch als fortschreitende Veränderung dieser Durchbiegung über den Versuchsverlauf (bis max. 10e5 LW) widerspiegelt. Bei der Beurteilung der dynamischen Belastbarkeit der Verbindungsart wurde darauf geachtet, dass die Funktionalität nach 100.000 Lastwechseln noch gewährleistet war bzw. die Durchbiegung nach geplantem Versuchende unter einem definierten kritischen Wert lag. Dieser wurde auf max. $5 \mathrm{~mm}$ zulässige Durchbiegung bei einer Stützweite von $1.500 \mathrm{~mm}$ festgelegt und hatte vorwiegend funktionale Gründe.

Tabelle 2: Ergebnisse der Kurzzeituntersuchungen zur Verbindungstechnik

\begin{tabular}{|c|c|c|c|}
\hline Versuch & $\begin{array}{c}\text { Mittelwert } \\
\text { erreichte } \\
\text { LW }\end{array}$ & $\begin{array}{c}\text { mittlere } \\
\text { Durchbiegung } \\
\text { [mm] (Endwert - } \\
\text { Anfangswert) }\end{array}$ & $\begin{array}{l}\text { hochgerechnete } \\
\text { Durchbiegung } \\
\text { auf eine Stütz- } \\
\text { weite von I = } \\
1.500 \mathrm{~mm}\end{array}$ \\
\hline SI 1 & 100.000 & 0,049 & 3,974 \\
\hline SI 2 & 100.000 & 0,116 & 9,405 \\
\hline EM 1 & 100.000 & 0,423 & 34,298 \\
\hline EM 2 & 53.479 & 1,231 & 99,812 \\
\hline HS & 11.235 & 0,547 & 44,352 \\
\hline QGB & 100.000 & 0,217 & 17,595 \\
\hline
\end{tabular}

Lediglich vier der sechs untersuchten Verbindungsmittel hatten die geforderte Mindestlastwechselzahl von 10e5 erreicht, sowohl die EM 2 als auch die zu Vergleichszwecken mit untersuchten HS versagten deutlich vor Erreichen der Mindestanzahl an Lastwechseln. Setzerscheinungen und Zerstörungen im Holzwerkstoff führten zum Versagen der Verbindung, Schädigungen am Verbindungsmittel konnten nur bei den EM 2 (Abbrechen der Zähne) festgestellt werden.

Als zweite Betrachtungsebene wurde die mittlere Durchbiegung zwischen Versuchsbeginn und -ende herangezogen. Hier konnten lediglich die SI 1 den Anforderungen genügen.

\subsection{Statische Untersuchungen}

Aufbauend auf den zuvor durchgeführten dynamischen Betrachtungen erfolgte ein statischer Ausreißversuch der entsprechenden Verbindungsmittel. Hierfür wurden in einer ersten Betrachtungsebene die zuvor dynamisch belasteten, jedoch noch intakten, Inserts in Richtung ihrer Verbindungsachse aus dem Holzwerkstoff herausgezogen. Der generelle Versuchsaufbau entspricht dem aus Abbildung $1 \mathrm{~b}$. 
Ziel der Untersuchung waren Erkenntnisse zum Einfluss des Strukturverhaltens des Holzwerkstoffs. Hierbei konnte festgestellt werden, dass zuvor dynamisch belastete Proben zum Teil höhere Auszugsfestigkeiten als unbelastete Proben aufwiesen. Besonders bei den Schraubinserts I und II konnte dieser Effekt beobachtet werden. Eine mögliche Erklärung dieses Verhaltens bieten lokale Verdichtungen im Holzwerkstoff, die linear zum Verdichtungsgrad ein Ansteigen der mechanischen Eigenschaften bewirken. Aus diesen Erkenntnissen heraus wurde als Vorzugsvariante das Insert vom Typ SI I gewählt, da sowohl im dynamischen als auch statischen Bereich hiermit die besten Ergebnisse erzielt werden konnten.

Der zweite Teil der statischen Versuchsreihen beinhaltet somit Versuche an Inserts dieses Typs unter Variation folgender Parameter:

- Bohrlochdurchmesser (Vgl. dazu mit Tabelle 3)

- Holzfeuchtegehalt bzw. klimatische Einflussbestimmung (Wassereinfluss, Wasserlagerung)

- Verleimung

Die gewählten Bohrlochdurchmesser orientierten sich am Schaftdurchmesser der jeweiligen Gewindegröße. Es erfolgten Versuche in Schritten zu 0,2 (M4 bis M8) mm bzw. 0,25 mm (M10). Die in Tabelle 3 angegebenen Untergrenzen sind der kleinste Durchmesser, bei dem ein Insert ohne schwerwiegende Strukturschädigungen in den Holzwerkstoffe eingebracht werden konnte.

Tabelle 3: Bohrungsdurchmesser der geprüften Inserts (SI I)

\begin{tabular}{|c|c|c|c|}
\hline $\begin{array}{c}\text { Gewin- } \\
\text { degröße }\end{array}$ & $\begin{array}{c}\text { Einschraublänge I } \\
{[\mathrm{mm}]}\end{array}$ & $\begin{array}{c}\text { Insertdurchmesser } \\
{[\mathrm{mm}]}\end{array}$ & $\begin{array}{c}\text { Bohrlochdurchmesser } \\
{[\mathrm{mm}] \text { und Schrittweite }}\end{array}$ \\
\hline M4 & 12 & 8 & 6,4 bis 7,$2 ; 4 \times 0,2 \mathrm{~mm}$ \\
\hline M5 & 12 & 10 & 8,2 bis 9,$0 ; 4 \times 0,2 \mathrm{~mm}$ \\
\hline M6 & 12 & 12 & 9,8 bis 10,$6 ; 4 \times 0,2$ \\
\hline M8 & 12 & 14 & 11,8 bis 12,$6 ; 4 \times 0,2$ \\
\hline M10 & 15 & 18,5 & 16,0 bis 16,$75 ; 3 \times 0,25$ \\
\hline
\end{tabular}

Der Einfluss des Holzfeuchtegehaltes wurde vergleichend zwischen trockenen (Holzfeuchte: ca. 7,5\%) und nassen (Holzfeuchte: ca. 49,3\%; nach 90h Wasserlagerung) Proben untersucht. Bei der Verleimung wurde auf einen Polyurethankleber nach DIN EN 204 [EN01] zurückgegriffen (D4 - kochwasserfeste Verleimung). Aus statistischen Gründen wurden jeweils sieben Einzelproben untersucht. In Abbildung 2 sind die Ergebnisse dieser Untersuchungen exemplarisch für den Gewindedurchmesser M6 aufgeführt. 


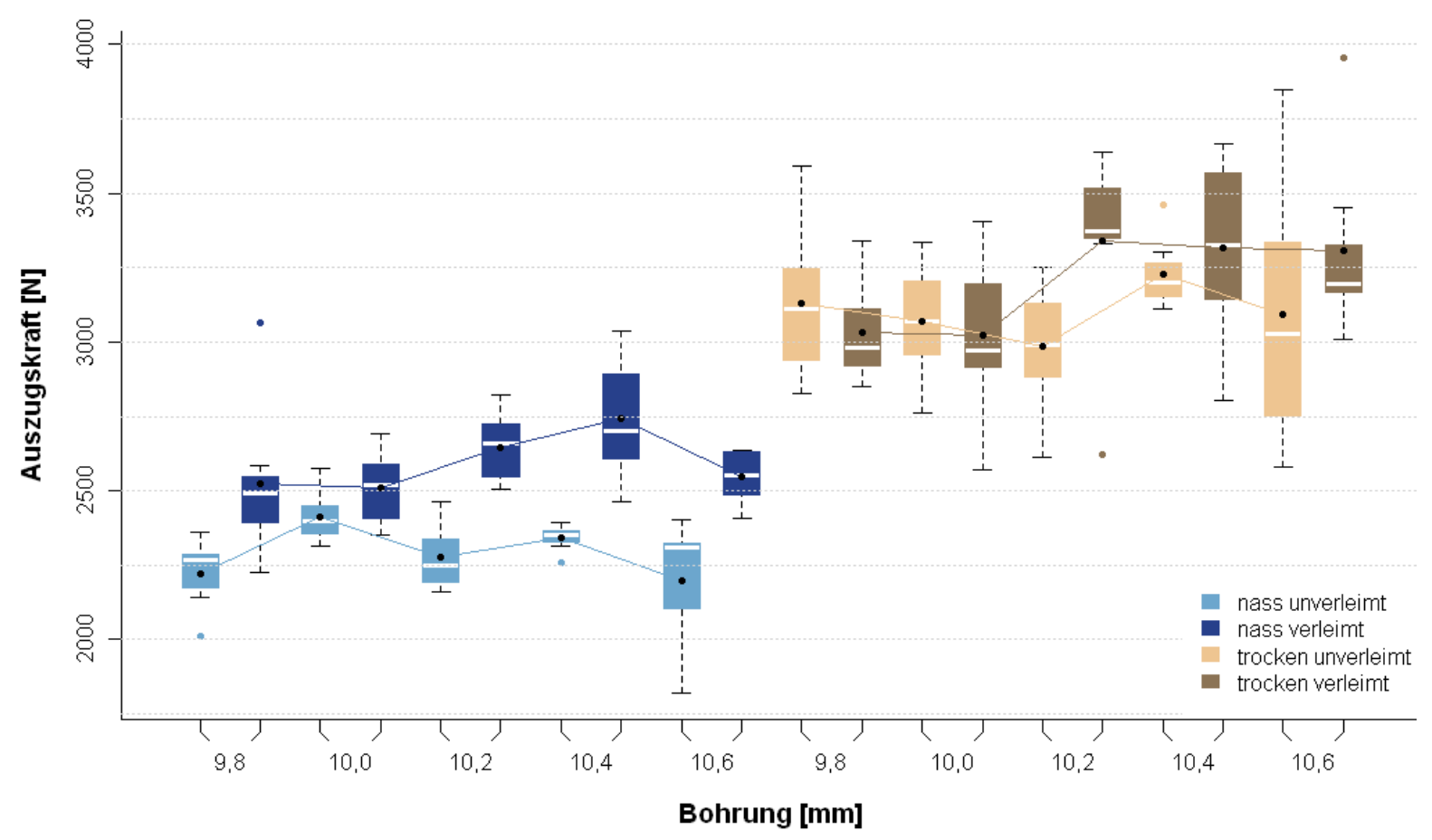

Abbildung 2: Mittelwertverlauf der Auszugskraft - M6

Die Ergebnisse der Untersuchungen zeigen neben den Werten für die maximale Belastbarkeit auch den Einfluss aller Parameter (Bohrlochdurchmesser, Feuchtegehalt des Holzes sowie Verleimung) auf die entsprechenden Kennwerte. Es wird deutlich, dass unter erhöhter klimatischer Belastung eine Verleimung durchaus sinnvoll ist, wohingegen unter geringer klimatischer Belastung nur ein geringer bzw. kein wirklicher Vorteil ableitbar ist. Der typische Kraft-Weg-Verlauf eines solchen statischen Zugversuchs kann Abbildung 3 entnommen werden.

Alle vier in Abbildung 3 dargestellten Verläufe zeichnen sich durch ein tendenziell nichtlineares Verhalten der Verbindung bis hin zum Erreichen der Maximalkraft aus. Diese kann jedoch nicht als Bemessungsgrundlage verwendet werden, da im praktischen Einsatzfall ein Auszugsweg des Inserts von mehr als $4 \mathrm{~mm}$ nicht vertretbar wäre und die Verbindung entsprechend eher versagen würde. Aus anwendungsorientierter Sicht ist demzufolge noch zu klären, bis zu welcher Kraft von einer elastischen Verformung in der Verbindung ausgegangen werden kann. 
a)

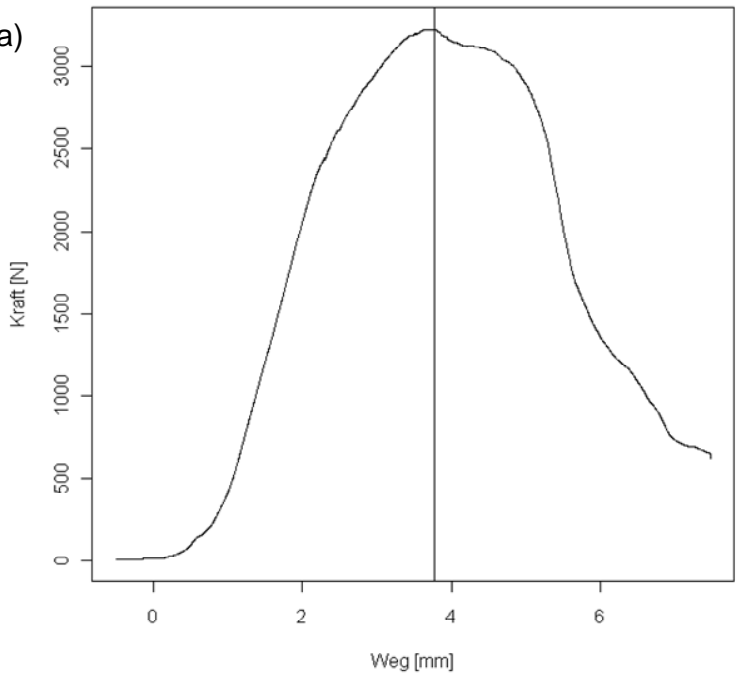

c)

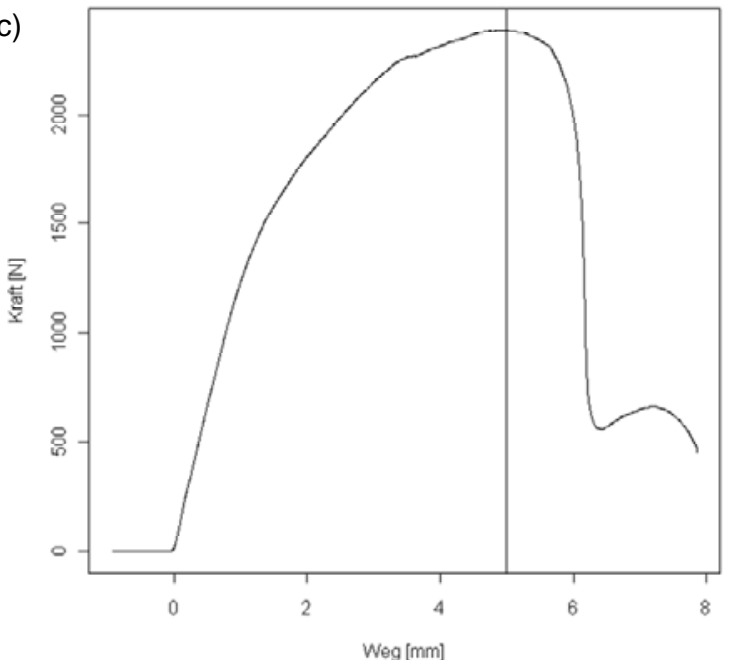

b)

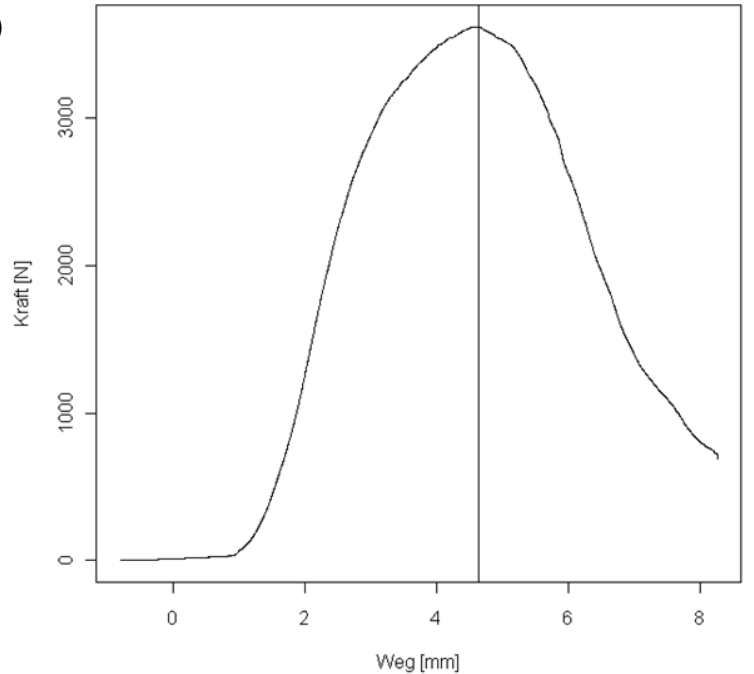

d)

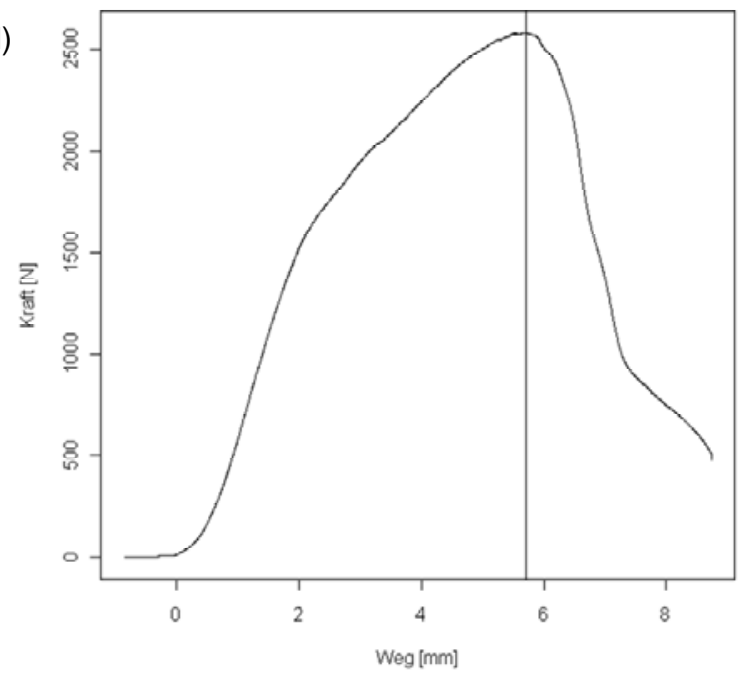

Abbildung 3: Charakteristische Kurvenverläufe der Zugversuche am Beispiel M6 - 10,4
a) trocken - unverleimt
b) trocken - verleimt
c) nass - unverleimt
d) nass - verleimt

\subsection{Dynamische Langzeituntersuchungen}

Die im vorherigen Abschnitt vorgestellten statischen Zugversuche bieten für die konstruktive Auslegung von Insertverbindungen nur geringen Informationsgehalt. Vor allem unter dynamischen Belastungen sind Dauerfestigkeitsgrenzen von übergeordneter Bedeutung. Das Ziel der nachfolgend vorgestellten dynamischen Langzeituntersuchungen lag somit darin, eine für die Auslegung nutzbare Aussage über die maximale Beanspruchung unter dynamischer Zuglast zu treffen. Der grundlegende Versuchsaufbau entspricht wiederum dem aus Abbildung $1 \mathrm{~b}$, jedoch diesmal unter schwellender dynamischer Zuglast. Folgende Parameter wurden hierbei untersucht:

- Probekörper analog zu den bisherigen (Abmessungen, Feuchtegehalt ca. 7,5\%)

- Inserttyp SI I; Bohrlochdurchmesser 10,4 mm; 5 Einzelproben je Versuchsreihe

- Unterkraft F = $100 \mathrm{~N}$, schwellende Last, sinusförmiger kraftgeregelter Versuch; Prüffrequenz: $20 \mathrm{~Hz}$ 
Die Oberkraft wurde relativ zum Mittelwert der Auszugskraft der statischen Untersuchungen (mittlere Auszugskraft: $F=3.231 \mathrm{~N}$ ) schrittweise (beginnend bei 100\% der statischen Auszugsfestigkeit) auf neue Probekörper aufgebracht und in Auswertung dessen über die Anzahl der Lastwechsel (als Funktion der Zeit) aufgezeichnet.

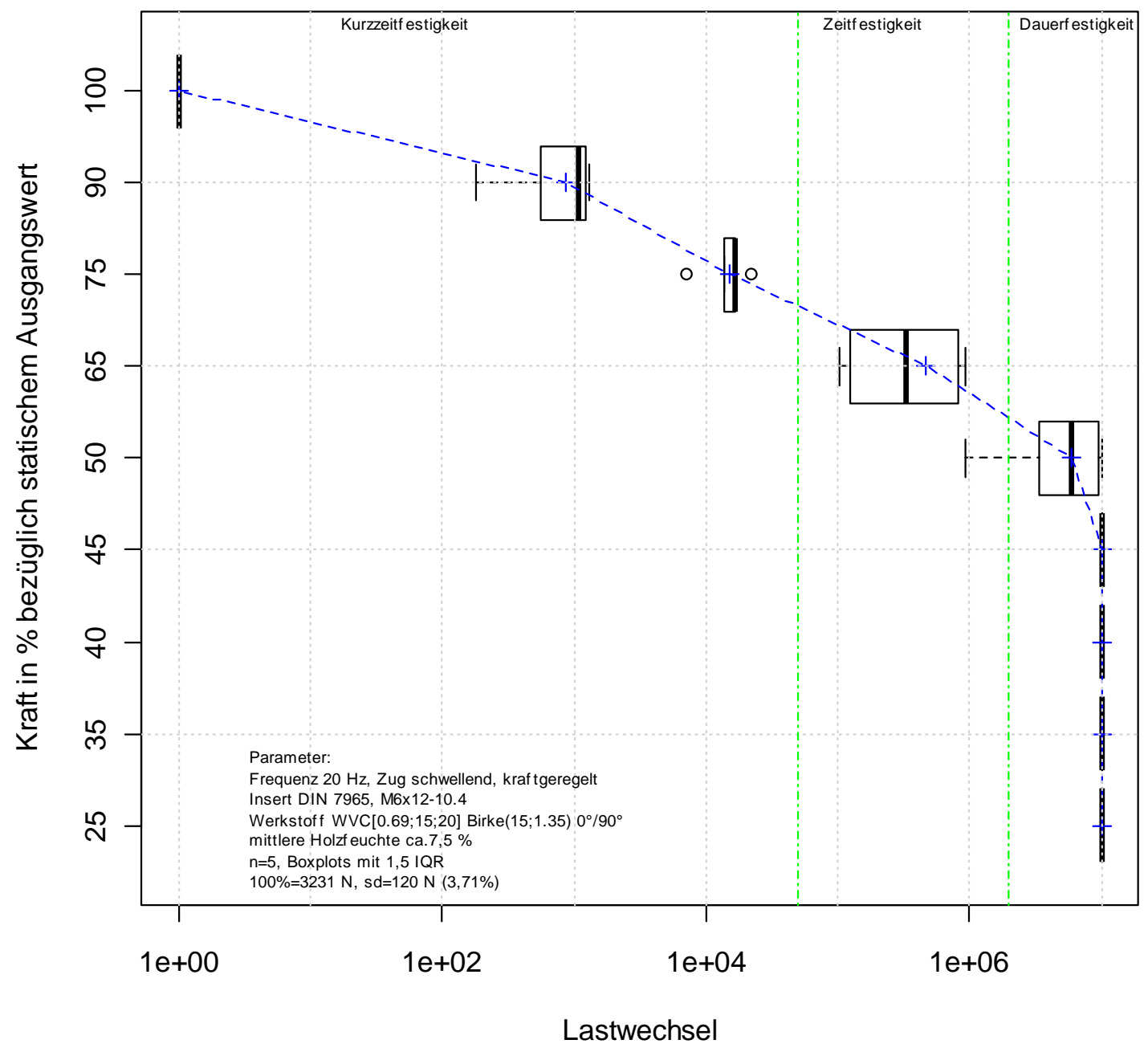

Abbildung 4: Dynamisches Langzeitverhalten unter schwellender Zuglast ( M6 - 10,4)

Bei der Durchführung und Auswertung der Versuche wurden die aus dem Maschinenbau typischen Grenzbereiche für Stahlwerkstoffe zur Kurzzeitfestigkeit bei $5 \times 10 e 4$ sowie der Zeitfestigkeit bei $2 \times 10 \mathrm{e} 6$ abgebildet. Ab einer erreichten Lastwechselanzahl von 10e7 kann von Dauerfestigkeit gesprochen werden. Die Ergebnisse dieser Untersuchungen sind Abbildung 4 zu entnehmen. Hierbei wird ersichtlich, dass bei ca. 50\% der statischen Auszugsfestigkeit der Bereich der Dauerfestigkeit unter dynamischen Lasten liegt. Im Anschluss an diese Pulserversuche wurden die Proben, die sich als dauerfest erwiesen haben, noch statisch ausgezogen und mit ungepulsten Proben verglichen. Hierbei konnte analog zu den Versuchen in Abschnitt 3.2 festgestellt werden, dass die dynamisch belasteten Proben höhere Auszugsfestigkeiten aufwiesen als unbelastete. Als Erklärung hierfür sind wahrscheinlich Setzerscheinungen und Verdichtungen im Grundwerkstoff zu sehen, die zu linearen Festigkeits- und Steifigkeitsanstiegen im Holzwerkstoff führen. 


\section{$4 \quad$ Funktionsmuster}

Anwendungsmöglichkeiten von Profilsystemen auf Basis von WVC in der technischen Logistik gibt es vor allem in Bereichen mit erhöhter Korrosion, beispielsweise der Grundstoff- und Kaliindustrie. Analog zu bestehenden Transporteinrichtungen erfolgte eine Umsetzung der in den Versuchen gewonnenen Erkenntnis in konkrete Anwendungen. Abbildung 5 zeigt den Prototypen eines Kettenförderers, dessen Grundgestell aus einem Hohlkammerprofil aus WVC besteht.

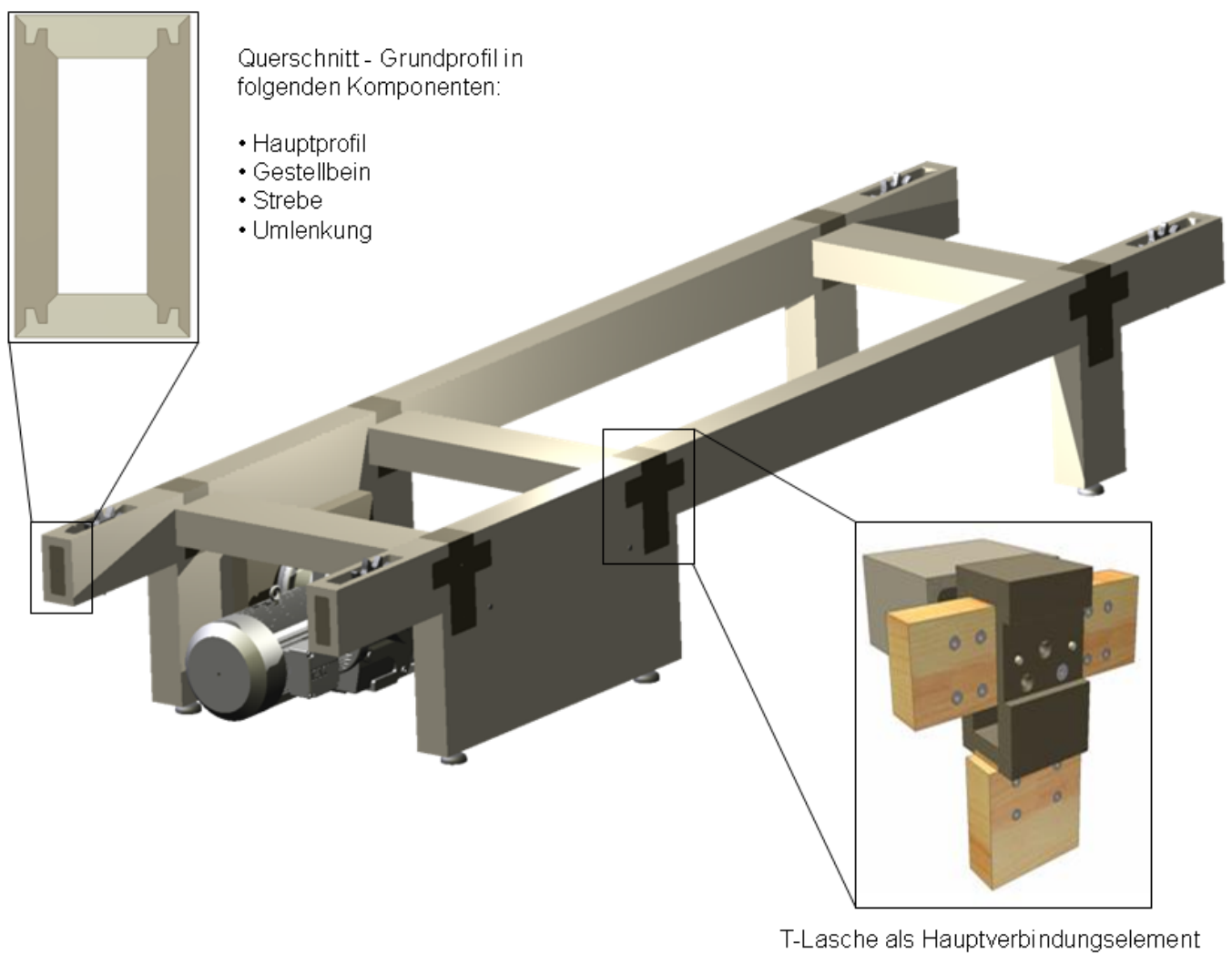

Abbildung 5: Prototyp eines Kettenfördersystems aus WVC (Darstellung ohne Zugmittel)

Dargestellt ist ein zweisträngiges Kettenfördersystem für den Stückguttransport. Das Grundprofil findet sich als Wiederholgruppe in praktisch allen Systemkomponenten des Gestells wieder. Als Hauptverbindungselement dient die T-Lasche, in der die zuvor untersuchten Gewindeeinsätze Anwendung finden. Unter Zuhilfenahme selbiger Verbindung ist eine modulare Bauweise problemlos realisierbar. Bei der Konzipierung des Prototyps wurde aus Gründen eines konstruktiven Korrosionsschutzes auf eine geschlossene Bauweise geachtet, der Anteil an freiliegenden Metallkomponenten wurde auf ein Mindestmaß reduziert. Die Zugmittelführung wird über eine (im Bild nicht dargestellte) Gleitschiene in Verbindung mit Kettenrädern realisiert, die Leertrumrückführung erfolgt im Inneren des Profils. Der Antrieb erfolgt über eine durchgängige Antriebswelle in Form eines Omega-Antriebs. Auch die Anbringung des Motors erfolgt unter Verwendung der untersuchten Inserts. Dabei konnten außer geringfügigen Setzerscheinungen selbiger im Grundwerkstoff keine Probleme festgestellt werden. Der Prototyp wurde über einen längeren Zeitraum im Reversierbetrieb untersucht. Zusätzlich zum generellen Funktionsverhalten wurden Schwin(c) 2010 Logistics Journal : Proceedings - ISSN 2192-9084 
gungs- und Geräuschmessungen durchgeführt. Gegenüber adäquaten Vergleichssystemen aus Stahl- und Aluminiumprofilen konnten aus technischer Sicht keine Nachteile festgestellt werden, aus wirtschaftlicher Sicht ergaben sich deutliche preisliche Vorteile. Für weiterführende Informationen zu modular aufgebauten Gestellsystemen in der Intralogistik aus Holz sei an dieser Stelle auf [EEN10a] sowie [EEN10b] verwiesen.

\section{$5 \quad$ Zusammenfassung und Ausblick}

Holz und Holzwerkstoffe sind übliche Materialien im Bauwesen, die unter statischen Gesichtspunkten klassifiziert und betrachtet worden sind. Modulare Gestellsysteme auf Basis dieser Werkstoffe erfordern eine Erweiterung der Betrachtungen auf dynamische Belastungen. Hierfür wurden spezielle Holzverbindungsmittel in Form von Gewindeeinsätzen aus Stahl ihrer Eignung entsprechend klassifiziert und sowohl statischen als auch dynamischen Kurz- und Langzeitversuchen unterzogen. Im Ergebnis dessen wurde eine Vorzugsform ausgewählt und in weiteren Analysen hinsichtlich ihrer Anwendbarkeit untersucht. In Ableitung dessen erfolgte die Konzipierung eines in der technischen Logistik nutzbaren Gestellsystems für einen Kettenförderer. Dieses Kettenfördersystem wurde umfangreichen Daueruntersuchungen unterzogen und seine prinzipielle Funktionalität konnte nachgewiesen werden. Das hierfür verwendete Basisgestell wurde unter geringfügigen konstruktiven Modifikationen in einer weiteren Förderanlage (Rollenbahn) umgesetzt, wobei die Erprobung dieses Systems gegenwärtig noch andauert. Die Auswertung der Versuche steht noch aus.

\section{Literatur}

[DIN65] DIN Deutsches Institut für Normung e.V.: DIN 7965: Einschraubmuttern (Schraubdübel). Berlin: Beuth Verlag, 1965.

[EEN10a] Eichhorn, Sven; Eckardt, Ronny; Nendel, Klaus: Modular aufgebaute intralogistische Gestellsysteme aus Holz - Teil I. In: f+h, Heft 4, S. 116118, 2010.

[EEN10b] Eichhorn, Sven; Eckardt, Ronny; Nendel, Klaus: Modular aufgebaute intralogistische Gestellsystem aus Holz - Teil II. In: f+h, Heft 5, S. 170171, 2010.

[EN01] DIN Deutsches Institut für Normung e.V.: Deutsche Fassung EN 204: Klassifizierung von thermoplastischen Holzklebstoffen für nichttragende Anwendungen. Berlin: Beuth Verlag, 2001.

[EN99] DIN Deutsches Institut für Normung e.V.: Deutsche Fassung EN 1382: Holzbauwerke - Prüfverfahren - Ausziehtragfähigkeit von Holzverbindungsmitteln. Berlin: Beuth Verlag, 1999.

[WS07] Wagenführ, André; Scholz, Frieder: Taschenbuch der Holztechnik. München: Fachbuchverlag Leipzig im Hanser-Verlag, 2007. 